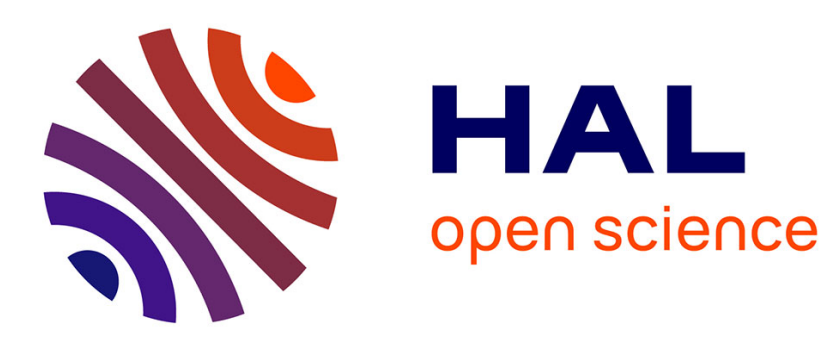

\title{
Wet Equivalent Potential Temperature and Enthalpy as Prognostic Variables in Cloud Modeling
}

\author{
Y. Pointin
}

\section{To cite this version:}

Y. Pointin. Wet Equivalent Potential Temperature and Enthalpy as Prognostic Variables in Cloud Modeling. Journal of the Atmospheric Sciences, 1984. hal-01975013

HAL Id: hal-01975013

https://hal.uca.fr/hal-01975013

Submitted on 8 Jun 2021

HAL is a multi-disciplinary open access archive for the deposit and dissemination of scientific research documents, whether they are published or not. The documents may come from teaching and research institutions in France or abroad, or from public or private research centers.
L'archive ouverte pluridisciplinaire HAL, est destinée au dépôt et à la diffusion de documents scientifiques de niveau recherche, publiés ou non, émanant des établissements d'enseignement et de recherche français ou étrangers, des laboratoires publics ou privés. 


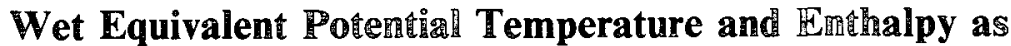 Prognostic Variahles in Cloud Modelining
}

\author{
Y. POINTIN \\ Laboratoire Associé de Météorologie Physique, Université de Clermont II, B.P. 45, 63170 Aubière, France
}

(Manuscript received 23 May 1983, in final form 30 September 1983)

ABSTRACT

\begin{abstract}
The bulk thermodynamic consequences of nonreversible phase changes and of the precipitation process are emphasized. Two predictive quantities are proposed, either of which can be used as a prognostic thermodynamic variable, instead of temperature or of potential temperature, in numerical models of convective clouds. The use of these new variables decreases the coupling between the dynamical and the thermodynamical equations and is shown to induce smaller errors under most circumstances involving physical processes such as the freezing of supercooled drops and the evaporation in undersaturated air. The respective advantages of both variables are discussed; the choice between them is shown to depend upon the phenomena to be simulated.
\end{abstract}

\section{1. 具踏oduction}

The numerical simulation of the flows which develop in a deep cumulonimbus cloud involves the solution of equations appropriate to a multiphase mixture of compressible gases, liquids and solids in which phase changes (condensation, evaporation, sublimation, freezing) take place. These equations must be able to describe as accurately as possible such aspects as the nonreversible phase changes (freezing of supercooled drops, evaporation in subsaturated air), the spatial transport of liquid water due to precipitation, and the nonhydrostatic part of the pressure which are some of the essential features of such flows. Therefore, the bulk thermodynamics of these phenomena should be accurately treated in a simple manner. However, the fundamental thermodynamical equation, obtained by applying an enthalpy conservation principle to a nonequilibrium open system, involves the rate of change of the partial pressure, the mixing ratio and the temperature of each phase, and is not very easy to integrate.

Although considerable attention has been paid to the thermodynamic equations (Betts, 1973; Iribarne and Godson, 1973; Dutton, 1976; Wilhelmson, 1977; Brook, 1978; Simpson, 1978; Paluch, 1979; Bolton, 1980; Tag, 1980; Lipps and Hemler, 1980) and to the equations appropriate to multiphase flows (Das, 1969; Tripoli and Cotton, 1981), it seems that all the terms which appear in the fundamental bulk thermodynamical equation have not yet been completely accounted for. The first goal of the present paper is to provide a complete formulation of the bulk thermodynamics of a convective cloud as well as to emphasize the thermodynamics of nonreversible phase changes.

In addition, two thermodynamical quantities are proposed for use as prognostic variables in numerical models instead of the mean temperature or one of the previously proposed potential temperatures (Betts, 1973; Wilhelmson, 1977; Paluch, 1979; Tripoli and Cotton, 1981). For cloud modeling, it is essential to make the right choice for this quantity since, as shown by several authors (Madden and Robitaille, 1970; Betts, 1973; Simpson, 1978), the maximum altitude of cumulus cloud predicted with the use of a thermodynamic equation is strongly dependent upon the conservative quantity used for the prediction. It is true that in threedimensional cloud modeling, other factors such as the subgrid parameterization scheme (turbulence closure), the dynamical scheme (hydrostatic versus nonhydrostatic, fully compressible versus anelastic), the boundary conditions and the initial conditions may be even more important. However, the bulk thermodynamical aspect of the flow is probably the first to consider when trying to avoid approximations made in earlier studies. These approximations are becoming more and more unnecessary due to the improvements in computers and in numerical methods of integration.

In Section 2, the basic equations are derived and justifications are provided for some approximations; in Section 3, numerical computations are performed in order to examine, in some extreme cases, the validity of the proposed "conservative" thermodynamical quantities. Analyses of the simulation results are given in Section 4.

\section{Formulation}

The cloudy air consists of dry air (index $d$ ), of water vapor (index $v$ ), of liquid water (index $l$ ) and of ice particles (index $i$ ). In this work, water vapor and dry 
air are treated as perfect gases with constant specific heats. Although molecular diffusion is important in estimating the rate of change of each drop radius, its effects on a characteristic length scale greater than a few decimeters are much less than the effects of turbulent diffusion which is not considered here. The mixing ratios and all the species' properties are defined as mean values over a volume which is of the order of a few cubic decimeters, i.e., large enough so that a large number of particles are in this volume, but small enough so that it can be considered homogeneous. The water vapor does not have homogeneous properties throughout the averaging volume considered since its temperature and mixing ratio vary around each drop due to the diffusive processes of heat and vapor. However, in general, the relative mass of water vapor directly affected by the drops is small and, therefore, the mean value of the water vapor temperature is taken as that of the dry air. The partial pressure of water vapor is generally below the saturated value at the corresponding temperature; condensation occurs as soon as this value is exceeded. The volume of the liquid water is neglected, compared to the specific volume of the water vapor (the error is less than 0.002), but the energy carried by the water or ice particles is fully considered.

The liquid water is assumed to consist of droplets which have a relative velocity $V_{l}$ in the air, a mean temperature $T_{l}$ and a concentration $\rho_{l}\left(\mathrm{~kg} \mathrm{~m}^{-3}\right)$ or a mixing ratio $r_{l}=\rho_{l} / \rho_{d}\left(\mathrm{~kg} \mathrm{~kg}^{-1}\right)$. The difference between the air temperature $T$ and the droplet temperature $T_{l}$ can be of the order of several degrees (Pruppacher and Klett, 1978) for drops of radius greater than $1 \mathrm{~mm}$ falling in subsaturated air. The time evolution of the drop temperature can be obtained from the heat budget of each drop; the adaptation time of the drop temperature to its steady-state value can be as large as $4.4 \mathrm{~s}$ for a drop of radius $1.35 \mathrm{~mm}$. Therefore, in the averaging volume, all drops of the same radius are assumed to have the same origin and identical properties which can be different from those of other drops. For simplicity, in the following derivation, all drops are assumed to have the same radius; this gives no loss of generality since generalization of the following equations to many classes of drops having different radius, temperatures and terminal velocities is straightforward and will be done in Section 3.

The solid phase is assumed to have a concentration $\rho_{i}$, a mixing ratio $r_{i}=\rho_{i} / \rho_{d}\left(\mathrm{~kg} \mathrm{~kg}^{-1}\right)$, a temperature $T_{i}$ and a mean relative velocity $\mathbf{V}_{i}$. Similar to the case of liquid particles, the generalization to many classes of ice particles is straightforward.

\section{a. The basic thermodynamic equation}

Dutton (1976) has considered the enthalpy variation during any change appearing in an open system and developed the first law of thermodynamics for such a case [see Tripoli and Cotton, 1981; their Eq. (1)]. Dut- ton's equation is written in terms of internal and external derivatives $\left(d_{i} \chi / d t\right.$ and $d_{e} \chi / d t$, respectively). The former derivative describes the change along the parcel path; the latter results from the energy flux into or out of the parcel and is here considered to be associated with the derivative along the relative motion of the precipitating particles alone. For example, and for any quantity $\chi$ (a list of symbols is given in the Appendix), we have

$$
\frac{d_{e}}{d t}\left(r_{l} \chi\right)=\mathbf{V}_{l} \cdot \nabla\left(\dot{r}_{l} \chi\right)
$$

or

$$
r_{l} \frac{d_{e}}{d t}(\chi)=r_{l} \mathbf{V}_{l} \cdot \nabla(\chi)
$$

while

$$
\frac{d_{e}}{d t}\left(r_{v} \chi\right)=\frac{d_{e}}{d t}\left(s_{d}\right)=0 .
$$

With this interpretation of Dutton's notation, the fundamental thermodynamical equation used in this paper reads, with the usual notations,

$$
\begin{aligned}
& \frac{d}{d t}\left(C_{p d} \ln T\right)-\frac{d}{d t}\left(R_{d} \ln P_{d}\right)+\frac{d}{d t}\left(r_{v} \frac{L_{l v}}{T}\right) \\
& -\frac{d}{d t}\left(r_{i} \frac{L_{i l}}{T}\right)+r_{v} \frac{d}{d t}\left(\frac{A_{l v}}{T}\right)-r_{i} \frac{d}{d t}\left(\frac{A_{i l}}{T}\right) \\
& +r_{T} C_{l} \frac{d}{d t} \ln T+r_{l} C_{l} \mathbf{V}_{l} \cdot \nabla \ln T_{l} \\
& -r_{i} \mathbf{V}_{i} \cdot\left[\nabla\left(\frac{A_{i l}}{T}\right)^{\prime}-C_{l} \nabla \ln T_{i}\right]=0
\end{aligned}
$$

where $d / d t$ is the time derivative following the air and water vapor (internal derivative in Dutton's notation), i.e.,

$$
d / d t=\partial / \partial t+\mathbf{u} \nabla
$$

where $\mathbf{u}$ is the air (and water vapor) velocity.

The term $r_{T} C_{l} d / d t \ln T$ in (1) represents energy change due to a water mass being advected with the fluid medium (Orville and Kopp, 1977). The term $r_{l} C_{l} V_{i} \cdot \nabla \ln T_{l}$ has already been obtained by Das (1969) and represents the cooling effect due to the downward flux, through the air parcel, of precipitating particles which warm up under a forced conduction process at the expense of the air parcel energy. The last term in (1) is partly due to the energy caught away by the precipitating ice particles and partly due to nonreversible change of entropy associated with the freezing process.

The affinity term $A_{i v}\left(A_{i l}\right)$ is defined as the change in the free enthalpy of water going from the liquid to the vapor state (from the ice to the liquid state) such that

$$
\begin{aligned}
& A_{l v}=h_{l}-T_{l} s_{l}-h_{v}+T_{v} s_{v} \simeq \bar{T}\left(s_{v}-s_{l}\right)-L_{l v}, \\
& A_{i l}=h_{i}-T_{i} s_{i}-h_{l}+T_{l} s_{l} \simeq \bar{T}_{i}\left(s_{l}-s_{i}\right)-L_{i l},
\end{aligned}
$$


where $\bar{T}\left(\bar{T}_{i}\right)$ is a mean temperature between $T$ and $T_{l}\left(T_{l}\right.$ and $\left.T_{i}\right)$. The last equalities hold because the phase changes occur at a constant total pressure (Dutton, 1976) and because the temperatures $T_{l}, T_{v}, T_{i}$ are not very different. These last equalities will be used to evaluate the affinity terms.

The affinity due to nonreversible processes can be assumed to be of the order of magnitude of the corresponding free enthalpy difference experienced along a reversible process going from the same initial state to the final one. Two different processes can be considered which start from liquid water at the temperature $T_{l}$ and lead to water vapor at the temperature $T$ and at the pressure $e_{v}$ (partial pressure of water vapor in the dry air). The first one evaporates the water at the temperature $T_{l}$, giving water vapor with the partial pressure $e_{s}\left(T_{l}\right)$ and then heats (or cools) the water vapor to the temperature $T$ while expanding it to the partial pressure $e_{v}$. The entropy change is therefore

$$
\begin{aligned}
\left(s_{v}-s_{l}\right)_{1}=L_{l v}\left(T_{l}\right) / T_{l}+C_{p v} & \ln \left(T / T_{l}\right) \\
& -R_{v} \ln \left[e_{v} / e_{s}\left(T_{l}\right)\right] .
\end{aligned}
$$

The second process warms (or cools) the liquid water to the temperature $T$, evaporates the water at this temperature, and expands the water vapor from the pressure $e_{s}(T)$ to the pressure $e_{v}$. The corresponding entropy change is

$\left(s_{v}-s_{l}\right)_{2}=\frac{L_{l v}(T)}{T}-C_{l} \ln \left(T / T_{l}\right)-R_{v} \ln \left[e_{v} / e_{s}(T)\right]$.

The use of the Clausius-Clapeyron's relation

$$
\frac{d e_{s}(T)}{d T}=\frac{L_{l v}(T) e_{s}(T)}{R_{v} T^{2}},
$$

together with Kirchhoff's law

$$
\frac{d L_{l v}}{d T}=C_{p v}-C_{l},
$$

shows that these two functions of $T$ and $T_{l}$ as given by (5) and (6) are identical. This enables the evaluation of the affinity $A_{w}$ by inserting (6) in (3), giving

$$
A_{l v} \approx-R_{v} T \ln \left[e_{v} / e_{s}(T)\right],
$$

where the second term in (6), which is an order of magnitude smaller than the third one, has been neglected and where the mean temperature $\bar{T}$ has been approximated by the air temperature $T$. This affinity term can be of the order of several percent of the latent heat of vaporization and should not be neglected in the simulation of flows in which drops evaporate in subsaturated air.

Similarly, the nonreversible process which consists in the freezing of supercooled droplets (at a temperature $T_{i}$ which can be as low as -20 to $-40^{\circ} \mathrm{C}$ ) gives an entropy change which can be assumed to be of the order of the change produced by the following hypothetical reversible process. In this latter process, the supercooled water is warmed up to the temperature $T_{0}=273.1 \mathrm{~K}$; freezing occurs and the ice is cooled back to the temperature $T_{i}$.

$s_{l}-s_{i}=C_{l} \ln \left(T_{l} / T_{0}\right)+L_{i l}\left(T_{0}\right) / T_{0}+C_{i} \ln \left(T_{0} / T_{i}\right)$.

In the case of freezing, the drop temperature $T_{l}$ cannot depart very much from the air temperature $T$ and, likewise, since the latent heat of freezing is small, the ice temperature $T_{i}$ is also close to the air temperature $T$. This affinity can be approximated by

$A_{i l} \approx T\left[\frac{L_{i l}\left(T_{0}\right)}{T_{0}}-\frac{L_{i l}(T)}{T}+\left(C_{l}-C_{i}\right) \ln \left(\frac{T}{T_{0}}\right)\right]$,

which is a function of the temperature $T$ alone. This affinity term can reach $20 \%$ of the latent heat of freezing and is identical to the expression proposed by Tripoli and Cotton (1981):

$$
A_{i l}=R_{v} T \ln \left[e_{i}(T) / e_{s}(T)\right],
$$

if the saturated vapor pressure over ice is related to the latent heat of sublimation by the Clausius-Clapeyron equation.

Equation (1), in which the affinity terms are evaluated from (9) and (11), is the fundamental thermodynamical equation used in this study. It relates the rate of change of temperature to those of the partial pressures and the mixing ratios under any circumstances (nonreversible phase changes, precipitation processes); equilibrium is not assumed. It differs from Tripoli and Cotton's (1981) fundamental equation [their Eq. (14)], by explicitly showing the influence of the precipitating particles [last terms in (1)] instead of using the internal and external derivatives. Eq. (1) shall be used as a norm to which the approximate equations will be compared.

In fact, Eq. (1) is not of practical use in numerical models since it is strongly coupled to the pressure tendency equation and to the microphysical equations predicting the value of the mixing ratios. It is, therefore, essential to define a quantity which has a much smaller variation along the particle path and is less dependent upon the fluctuations of partial pressures and of mixing ratios than the temperature. Two such quantities are defined in the next two subsections.

Tripoli and Cotton (1981) have already considered several approximations and proposed an ice-liquid potential temperature (ILPT) defined by

$$
\theta\left[1+\frac{\left.L_{l v}\left(T_{0}\right)\left(r_{i}+r_{l}\right)+L_{i l}\left(T_{0}\right) r_{i}\right]}{C_{p d} \max (T, 253 .)}\right]^{-1},
$$

where the potential temperature $\theta$ is given by Poisson's equation

$$
\theta=T\left(P_{0} / P\right)^{R_{d} / C_{p d}}
$$


and $P_{0}=1000 \mathrm{mb}$. It will be shown in the third part of this paper that, in some extreme situations, this iceliquid potential temperature leads to errors of up to $5 \mathrm{~K}$ in the evaluation of the potential temperature of a moist parcel and is not the most accurate quantity to be considered.

\section{b. Wet equivalent potential temperature (WEPT)}

In order to derive a new potential temperature, two approximations can be considered.

- The first approximation involves the replacement of the term

$$
r_{v} \frac{d}{d t}\left(\frac{A_{l v}}{T}\right) \text { by } \frac{d}{d t}\left(r_{v} \frac{A_{l v}}{T}\right)
$$

This replacement induces no errors in two cases:

1) When $A_{l v} \approx 0$; this occurs during the condensation since the supersaturation is very small in clouds and $e_{\mathrm{v}} \approx e_{s}(T)$.

2) When $r_{v}$ is constant; this occurs when the moist air does not contain liquid or ice particles.

- Similarly, the second approximation replaces

$$
r_{i} \frac{d}{d t}\left(\frac{A_{i l}}{T}\right) \text { by } d\left(r_{i} \frac{A_{i l}}{T}\right) .
$$

The magnitude of the terms involved by these approximations is only a few percent of the magnitude of the dominant terms in (1).

With these two approximations, the thermodynamic equation is approximated by

$$
\begin{gathered}
C_{p t} \frac{d \ln T}{d t}-R_{d} \frac{d \ln P_{d}}{d t^{*}}+\frac{d}{d t}\left\{\left[L_{1}(T) r_{v}-L^{\prime}(T) r_{i}\right] / T\right\} \\
+\left\{r_{i} \mathbf{V}_{i}\left[C_{l}-\frac{L_{i l}}{T}\right]+C_{l} r_{l} \mathbf{V}_{l}\right\} \cdot \nabla \ln T=0,
\end{gathered}
$$

where

$$
\begin{aligned}
C_{p t} & =C_{p d}+r_{T} C_{l}, \\
L_{1}(T) & =L_{l v}(T)-R_{v} T \ln \left[e_{v} / e_{s}(T)\right], \\
L^{\prime}(T) & =L_{i l}(T)+A_{i l}(T) .
\end{aligned}
$$

One consequence of this equation is that the wet equivalent potential temperature (WEPT), defined by

$$
\theta_{q}=T\left(P_{0} / P_{d}\right)^{R_{d} / C_{p t}} \exp \left\{\left[L_{!}(T) r_{v}-L^{\prime}(T) r_{i}\right] /\left(T C_{p t}\right)\right\},
$$

has a rate of change which follows the equation

$$
\begin{aligned}
C_{p t} \frac{d \ln \theta_{q}}{d t} & =\ln \left(T / \theta_{q}\right) C_{l} \frac{d r_{T}}{d t} \\
& -\left[r_{i} \mathbf{V}_{i}\left(C_{l}-\frac{L_{i l}}{T}\right)+C_{l} r_{l} \mathbf{V}_{l}\right] \cdot \nabla \ln T
\end{aligned}
$$

where the first term on the right-hand side results from the variation of $C_{p t}$ within the exponent. The change in the total mixing ratio $r_{T}$ is due to precipitations and is easily shown to be given by (Das, 1969)

$$
\frac{d r_{T}}{d t}=\nabla \cdot\left(\rho_{l} \mathbf{V}_{l}+\rho_{i} \mathbf{V}_{i}\right) / \rho_{d}
$$

Therefore, the wet equivalent potential temperature $\theta_{q}$ is essentially constant (within the two approximations shown in Section 2b) during any transformation even when it involves phase changes, except when precipitating particles are present. Paluch (1979) has used a similar quantity (without the ice phase) in an experimental study of the entrainment in clouds; however, two different expressions were found to be necessary, corresponding to undersaturated and saturated conditions. Keeping the affinity of condensation $A_{l v}$ in the thermodynamical equation makes it possible to define this unique expression [Eq. (15)].

As shown in Section 3, it is easy to set up an iterative scheme in order to compute the temperature $T$ and the vapor mixing ratio from the values of the wet equivalent potential temperature. However, the main difficulty with its use in numerical models lies in the fact that its evaluation involves the lengthy computations of exponential, logarithmic and power functions.

\section{c. Wet equivalent enthalpy (WEE)}

A second approach to the simplification of the basic thermodynamical equation (1) is as follows.

On elimination of the partial pressure of dry air through the state equations, (1) can be exactly rewritten as

$$
\begin{aligned}
\left(C_{p d}+r_{T} C_{l}\right) & \frac{d T^{\prime}}{d t}-\frac{\left(1+r_{T}\right)}{\rho_{T}} \frac{d P}{d t}+\frac{d}{d t}\left(L_{l v}(T) r_{v}\right) \\
& -T \frac{d}{d t}\left[\frac{L^{\prime}(T) r_{i}}{T}\right]+A_{i l} \frac{d r_{i}}{d t} \\
+ & {\left[r_{i} \mathbf{V}_{i}\left(C_{l}-\frac{L_{i l}}{T}\right)+C_{l} r_{l} \mathbf{V}_{l}\right] \cdot \nabla T=0, }
\end{aligned}
$$

where $\rho_{T}=\rho_{d}\left(1+r_{T}\right)$ is the total density. This equation can be further modified in order to eliminate the water vapor mixing ratio in favor of the total mixing ratio, i.e.,

$$
r_{v}=r_{T}-r_{l}-r_{i}
$$

Some straightforward algebra leads to

$$
\begin{array}{r}
C_{p d}\left(1+\epsilon^{\prime} r_{T}\right) \frac{d T}{d t}-\frac{\left(1+r_{T}\right)}{\rho_{T}} \frac{d P}{d t}-\frac{d}{d t}\left[L_{l v}\left(r_{l}+r_{i}\right)\right] \\
-T \frac{d}{d t}\left[\frac{L^{\prime}(T) r_{i}}{T}\right]+A_{i l} \frac{d r_{i}}{d t}+L_{l v}(T) \frac{d r_{T}}{d t} \\
+\quad\left[r_{i} \mathbf{V}_{i}\left(C_{l}-\frac{L_{i l}}{T}\right)+C_{l} r_{l} \mathbf{V}_{l}\right] \cdot \nabla T=0
\end{array}
$$


where $\epsilon^{\prime}=C_{p v} / C_{p d}$ is the ratio of the specific heat at constant pressure of water vapor to that of dry air.

This energy equation shows that the wet equivalent enthalpy (WEE) defined by

$$
\begin{aligned}
H 1=C_{p d} & {\left[1+\left(\epsilon^{\prime}-1\right) r_{T}\right] T-g z } \\
& -\left[L_{l v}(T)\left(r_{l}+r_{i}\right)+T_{0} \frac{L^{\prime}(T) r_{i}}{T}\right]\left(1-r_{T}\right)
\end{aligned}
$$

is almost conserved under any adiabatic process involving nonreversible phase changes without precipitating particles. Indeed, the rate of change of the wet equivalent enthalpy is approximately given by

$$
\begin{aligned}
\frac{d H 1}{d t} & \approx-\left[r_{i} \mathbb{V}_{i}\left(C_{l}-L_{i l} / T\right)+C_{l} r_{l} \mathbb{V}_{l}\right] \cdot \nabla T\left(1-r_{T}\right) \\
& +\frac{1}{\rho_{T}}\left[\frac{d}{d t}(P)-\rho_{T} g w\right] \\
& +\frac{d r_{T}}{d t}\left[C_{p d} T\left(\epsilon^{\prime}-1\right)-L_{l v}\left(1-r_{T}-r_{l}-r_{i}\right)\right] .
\end{aligned}
$$

The first term on the right-hand side is due to the energy caught by the precipitating particles which come from higher altitudes with a lower temperature and fall through the moist air exchanging energy with the air parcel. The second term vanishes in hydrostatic atmospheres but should not be neglected inside deep convective clouds. The third term arises from the fact that the wet equivalent enthalpy is an extensive quantity which must vary when the total mixing ratio changes. The wet equivalent enthalpy is at most quadratic in the various parameters and, as shown in the third part, it is easy to set up an iterative scheme in order to solve for the parcel temperature knowing its enthalpy, its altitude, and the rain, ice, graupel and total mixing ratios.

\section{Purmerical evaluation of the errors using a Lagran- gian model}

Following Tripoli and Cotton (1981), numerical computations are carried out in order to estimate the errors involved in the simulation of convective flows using the two different approximations considered in this work. The basic equation considered in this paper, namely (1), in which the affinity terms are evaluated from (9) and (11), is solved for the temperature at the next level $[T(P+\Delta P)]$. This temperature is considered as the reference value to which the parcel temperatures, deduced from the potential temperatures or enthalpy, are compared. The differences between the corresponding potential temperatures indicate the errors that can be made when any of these quantities (potential temperatures or enthalpy) is used as a predictive quantity in a numerical model instead of using the complete basic equation.
Three tests have been conducted under the following general conditions. The parcel rises in a hydrostatic atmosphere with a vertical velocity which is a function of the total pressure alone given by

$$
w(P)=w_{\max } \sin \left[\left(P_{1}-P\right) \pi / P_{1}\right],
$$

where $w_{\max }=10 \mathrm{~m} \mathrm{~s}^{-1}$ and $P_{1}=1100 \mathrm{mb}$.

The microphysical structure of the parcel is characterized by five mixing ratios of the different water species: $r_{v}$ for the water vapor, $r_{c}$ for the cloud droplets, $r_{r}$ for the precipitating drops, $r_{i}$ for the ice crystals and $r_{h}$ for the precipitating graupel. The microphysical equations are similar to those used by Tripoli and Cotton and imply no supersaturation. Condensation/ evaporation of cloud droplet $\left(r_{c}\right)$ occurs whenever the partial pressure of water vapor becomes larger/smaller than its saturated value. The function $e_{s}(T)$ is obtained as the result of the analytical integration of the ClausiusClapeyron equation [(7)] in which the latent heat of vaporization is a linear function of the temperature satisfying (8).

In each case, the parcel starts its ascent from 1000 $\mathrm{mb}$ where it has a temperature of $310 \mathrm{~K}$ and a total mixing ratio of 0.02 . It rises up to $100 \mathrm{mb}$ and then descends back to the $1000 \mathrm{mb}$ level with the negative vertical velocity given by (23) with $w_{\max }=-10 \mathrm{~m} \mathrm{~s}^{-1}$. During the descent, the rain and graupel particles evaporate slowly according to the microphysical equations.

The integration scheme is based on the iterative solution of the implicit equation

$$
\chi(P+\Delta P)=\chi(P)-\Delta P \frac{d \chi}{d t}(\bar{P}, \bar{T}, \bar{r}) /\left(\bar{\rho}_{T} g \bar{w}\right),
$$

where $\chi$ stands for any parameter and where quantities with overbars are evaluated as mean values between the values at the upper and at the lower level. The pressure increment $\Delta P$ is adjusted so that the change in mixing ratios remains between $10^{-5}$ and $5 \times 10^{-5}$ and the corresponding time step is less than $10 \mathrm{~s}$.

In the discretization of the rate equations, the terms involving the time derivatives are all evaluated as

$$
\begin{gathered}
A(P, T, r) \frac{d \chi}{d t} \approx \frac{1}{2}\left[\frac{\chi(P+\Delta P)-\chi(P)}{\Delta P}\right][A(P+\Delta P, \\
T+\Delta \mathrm{T}, r+\Delta r)+A(P, T, r)]\left(\bar{\rho}_{T} g \bar{w}\right),
\end{gathered}
$$

where $A(P, T, r)$ is any function. This numerical scheme implies that the condensation adjustment step is part of the iterative solution of the resulting implicit equation. The iteration stops whenever the new estimate of the temperature is closer than $0.001 \mathrm{~K}$ to the last estimate. The integration from 1000 to $100 \mathrm{mb}$ takes $600-900$ steps with a mean pressure increment $\Delta P$ $\approx 0,5 \mathrm{mb}$. The total simulated duration of the parcel ascent (or descent) is about $25 \mathrm{~min}$ with the vertical velocity specified by (23). 
For each parcel predictive quantity (potential temperatures or enthalpy), the same semi-implicit integration scheme of its rate of change equation [(16) and (22)] is set up in order to predict its new value at the level $P+\Delta P$. The value of the ice-liquid potential temperature changes only under the influence of liquid water and ice unloading due to its extensive property (Tripoli and Cotton, 1981). In each case, the values of the rain, ice, graupel and total mixing ratios are assumed to be given by the referenced values obtained during the integration process of the basic equation. The values of the temperature, the cloud water and water vapor mixing ratios, the air density and the altitude are obtained each time as results of the inversion of each predictive quantity. Educated guess values of the above parameters are assumed, except the temperature, which is deduced from the value of the predictive quantity. A new estimate of the other parameters is deduced from the temperature, the saturated vapor mixing ratio and all the "referenced" values.

Three cases have been considered in the numerical simulations:

CASE I. The terminal velocity of rain and graupel particles is set equal to zero and the total mixing ratio is constant. In this case, all the predictive quantities (potential temperatures or enthalpy) happen to stay constant. This case will tend to overestimate the errors since the condensate mixing ratio can reach a value of 0.02 which is seldom reached inside real clouds.

CASE II. The terminal velocities are functions of the mixing ratios but the total mixing ratio remains constant. Here terms like $V_{h} \cdot \nabla T$ are taken to be identical in all four integrations. The comparison of this case to case I will give an indication of the order of magnitude of the last two terms of (1) which have usually been neglected previously (except for Das, 1969). These terms describe the cooling of the air due to the effect of the downward flux of cooler precipitating particles. Since the precipitation mixing ratios are too high due to the absence of fallout mechanisms, the consequences of these terms will be slightly overestimated.

CASE III. The total mixing ratio decreases due to the precipitation of rain and graupel particles, which is modeled by the arbitrary equations

$$
\left.\begin{array}{l}
\frac{d r_{r}}{d t}=-r_{r} / \tau \\
\frac{d r_{h}}{d t}=-r_{h} / \tau
\end{array}\right\},
$$

where $\tau=2000 \mathrm{~s}$ is a relaxation time chosen to be larger than the total time for the parcel rise or descent. In this case, the error should be underestimated since no processes of rain accumulation have been considered and the rain and graupel mixing ratios can be very small.

\section{Results of the simulations}

a. Case I

The potential temperature errors are defined as the difference between the potential temperatures which are obtained from the use of the three predictive quantities $\left(\theta_{i l}, \theta_{q}, H_{1}\right)$ and the potential temperature of the parcel obtained as the result of the integration of the basic equation (1). These errors are shown, for case I, in Fig. 1a by the curves labeled 1-3 as functions of the parcel pressure during the rise of the parcel. The value of each mixing ratio is shown in Fig. $1 b$, as a function of the pressure, by the vertical distance between the two curves enclosing each domain during the rise of the parcel. Figs. $2 a$ and $2 b$ show similar results for a descending parcel. The lowest curves in Figs. $\mathrm{lb}$ and $2 \mathrm{~b}$ correspond to the water vapor mixing ratio and the dotted curve corresponds to their saturated values. During the rise of the parcel, these two curves are identical (Fig. 1b) while, during the descent (Fig. 2b), the relative humidity is indicated by the ratio of the distances between each curve and the axis, and can be as low as $15 \%$ for any pressure greater than $800 \mathrm{mb}$ in case I. The total mixing ratio, which is indicated by the vertical distance between the highest curve in Figs. $1 \mathrm{~b}$ and $2 \mathrm{~b}$ and the axis, can be seen to stay constant in case $\mathrm{I}$.

As shown in Fig. $1 \mathrm{~b}$ during the parcel rise, the small droplets appear as soon as the parcel becomes saturated at about $850 \mathrm{mb}$, are changed by autoconversion into precipitating drops for pressures below $650 \mathrm{mb}$, and are completely frozen into small crystals for pressures below $180 \mathrm{mb}$. Precipitating drops grow by accretion of small droplets and by self-coalescence and start to freeze for pressures below $300 \mathrm{mb}$. Ice crystals, which originate from the freezing of small droplets, are accreted by raindrops and by precipitating graupel.

As shown in Fig. 2b, during the parcel descent, the

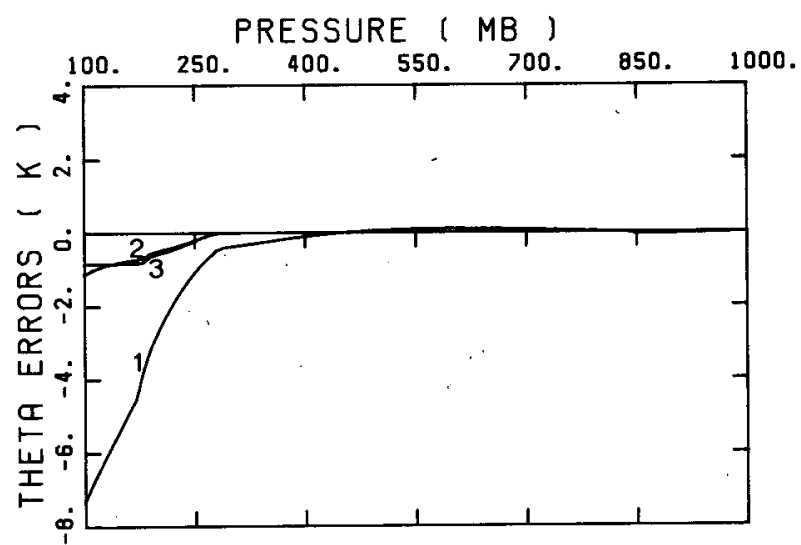

FlG. 1a. Potential temperature errors (K) for 1 , the ice-liquid potential temperature, 2 , the wet equivalent potential temperature, 3 , the wet equivalent enthalpy, during parcel rise (from right to left) in case $I$ as functions of the total pressure. 


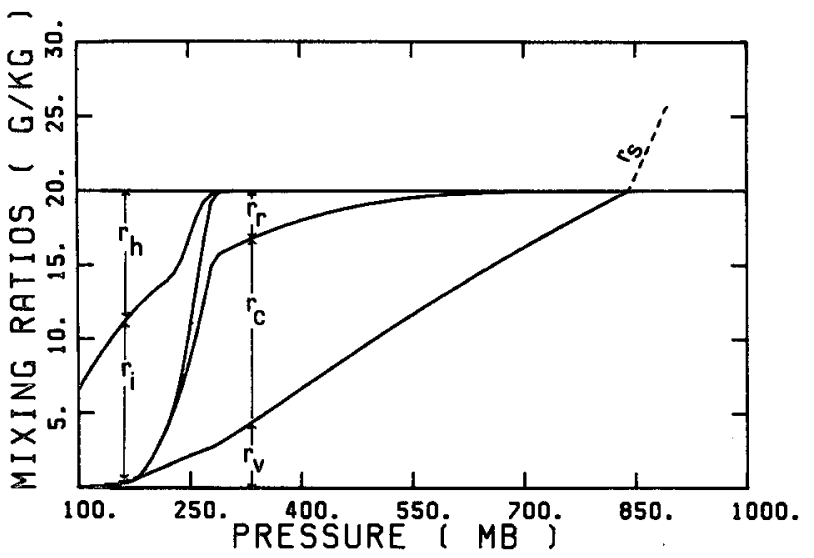

FlG. 1b. Mixing ratios versus pressure during parcel rise (from right to left) in case I.

precipitating graupel continue to accrete the small ice crystals and both species start to sublimate in the unsaturated air. For pressure higher than $320 \mathrm{mb}$, ice crystals followed by precipitating graupel start melting into small droplets which evaporate instantaneously and into precipitating drops which evaporate slowly in the unsaturated air. The water vapor mixing ratio increases up to 0.0085 at $1000 \mathrm{mb}$ and precipitating drops reach the ground.

During the first part of the parcel rise shown in Figs. $1 \mathrm{a}$ and $\mathrm{b}$, the parcel temperature deduced from each conserved quantity is remarkably close to the "reference value" until the parcel reaches $300 \mathrm{mb}$ (Fig. 1a) when ice particles appear (Fig. Ib). The potential temperature error levels off at a value of about $1 \mathrm{~K}$ for the temperature deduced from the wet equivalent potential temperature or the wet equivalent enthalpy, but reaches $-7 \mathrm{~K}$ for the ice-liquid potential temperature.

During the parcel descent (Figs. 2a and b), the temperature errors for two predictive quantities increase below $300 \mathrm{mb}$ due to the evaporation of raindrops in

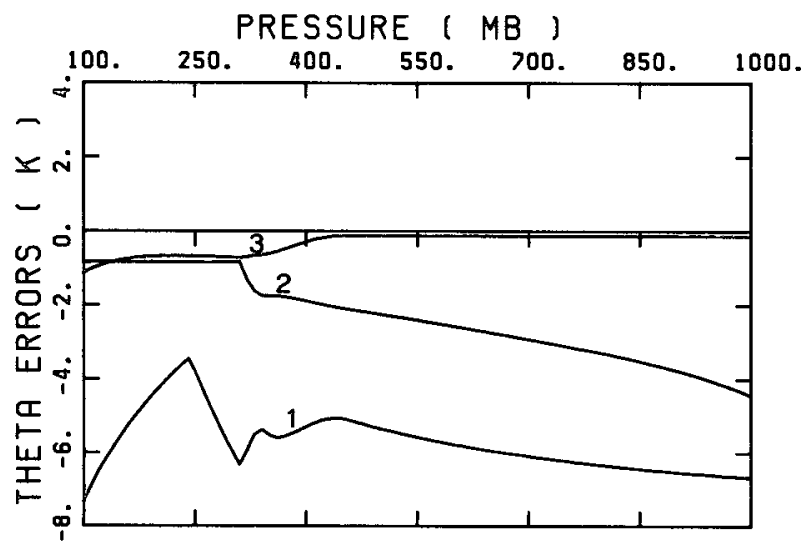

FIG. 2a. As in Fig. 1a except during parcel descent (from left to right) in case I.

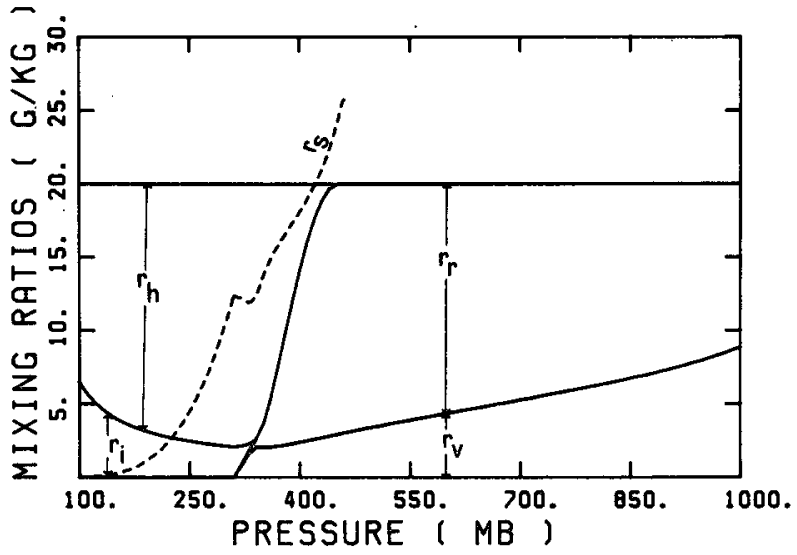

FIG. 2b. As in Fig. 1b except during parcel descent (from left to right) in case $I$.

the subsaturated air. However, the potential temperature deduced from the wet equivalent enthalpy becomes closer to the reference potential temperature and the errors become as low as $0.12 \mathrm{~K}$, showing that this quantity can take into account the thermodynamic effects due to all the nonreversible phase changes. The wet equivalent and the ice-liquid potential temperatures can lead to errors of several degrees in the determination of the parcel potential temperature.

The numerical errors created by the integration of (1), (16) and (22) accumulate during the ascent and the descent. However, these numerical errors can be estimated by the wet equivalent potential errors and the wet equivalent enthalpy errors below $300 \mathrm{mb}$ during the parcel rise since, in this case, the approximations are exact. Fig. 1a shows that these numerical errors are below $0.05 \mathrm{~K}$ between 1000 and $300 \mathrm{mb}$.

\section{b. Case II}

The curves corresponding to the parcel rise (not shown) are almost identical to those of Figs. 1a and $1 \mathrm{~b}$, since precipitating particles appear only above 600 $\mathrm{mb}$. Figure 3a concerns the parcel descent and shows that, beside the curve relative to the ice-liquid potential temperature (curve 1) which rises up to $5 \mathrm{~K}$, the potential temperature errors are almost identical to those of case I. Indeed, the rate of change equations for the wet equivalent potential temperature and for the wet equivalent enthalpy includes the cooling effect due to precipitation while, in this case, the ice-liquid potential temperature does not change since the total mixing ratio is kept constant. The mixing ratios are shown in Fig. $3 b$ for the parcel descent. These curves indicate that the humidity is larger in case II than in case I for any pressure larger than $300 \mathrm{mb}$. This fact is due to the cooling of the air induced by the downward flux of precipitating particles (cooler particles) through the parcel. 


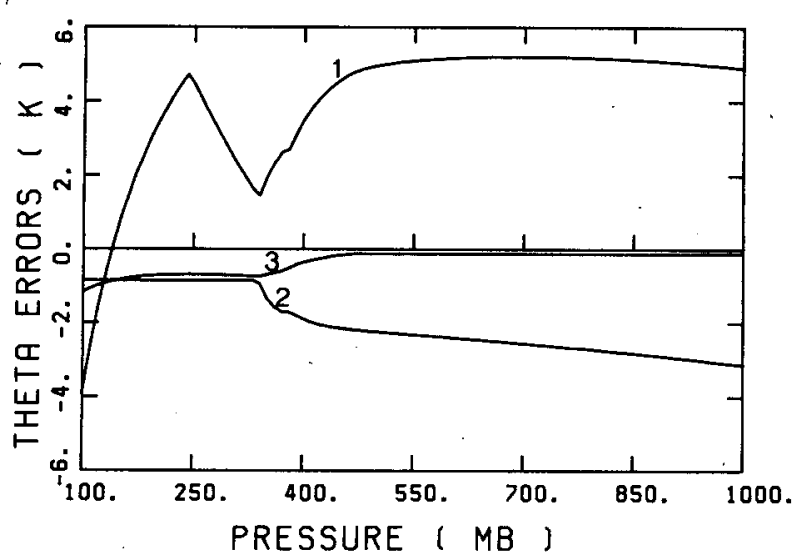

FIG. 3a. As in Fig. 1a except during parcel descent (from left to right) in case II.

\section{c. Case III}

The curves of the potential temperature errors as functions of pressure (not shown for the parcel rise and shown in Fig. 4a for the parcel descent) are almost identical to the corresponding curves for case I, even if the magnitude of the errors is slightly less in this case. The total mixing ratio decreases from $20 \mathrm{~g} \mathrm{~kg}^{-1}$ to $16.8 \mathrm{~g} \mathrm{~kg}^{-1}$ during the parcel rise and, as shown in Fig. $4 \mathrm{~b}$, decreases further to $11.4 \mathrm{~g} \mathrm{~kg}^{-1}$ during the parcel descent. Due to the inclusion of a precipitation process, the mixing ratio for precipitating drops and ice particles are smaller in this case than in case $I$ or II. This implies that the potential temperature errors which are mainly caused by the nonreversible evaporation process of ice and rain particles and by the precipitation process are less important than in case I or II.

For the choice of a predictive quantity to be used in the simulation of atmospheric flows, two aspects

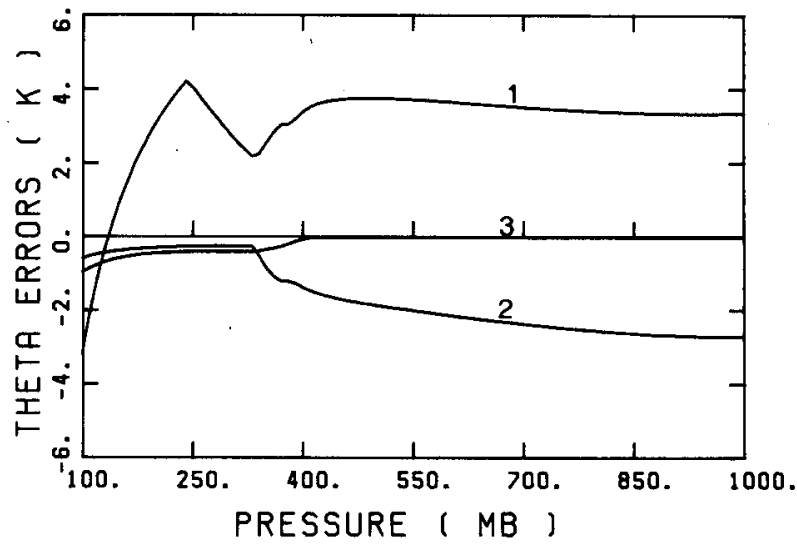

FiG. 4a. As in Fig. 1a except during parcel descent (from left to right) in case III.

must be considered. The first is the errors of the simulation and has been treated above. The second aspect is concerned with the coupling between the thermodynamical, microphysical and kinematical (continuity) equations. This coupling is the weakest when the change in the predictive quantity is small; that is, when its new value is close to its old value during the integration.

The changes in the predictive quantities during the parcel descent are shown in Fig. 5 as functions of the pressure. The change in the wet equivalent enthalpy is expressed in kelvins obtained by dividing it by the specific heat $C_{p d}$ of dry air at constant pressure. The largest values of the change in the predictive quantities are approximately $25.4 \mathrm{~K},-0.7 \mathrm{~K}$ and $16 \mathrm{~K}$ for the ice-liquid potential temperature, for the wet equivalent potential temperature and for the wet equivalent enthalpy, respectively. Curve 2 in Fig. 5 suggests that, in this case, with very small errors, the wet equivalent potential temperature can be assumed to be constant

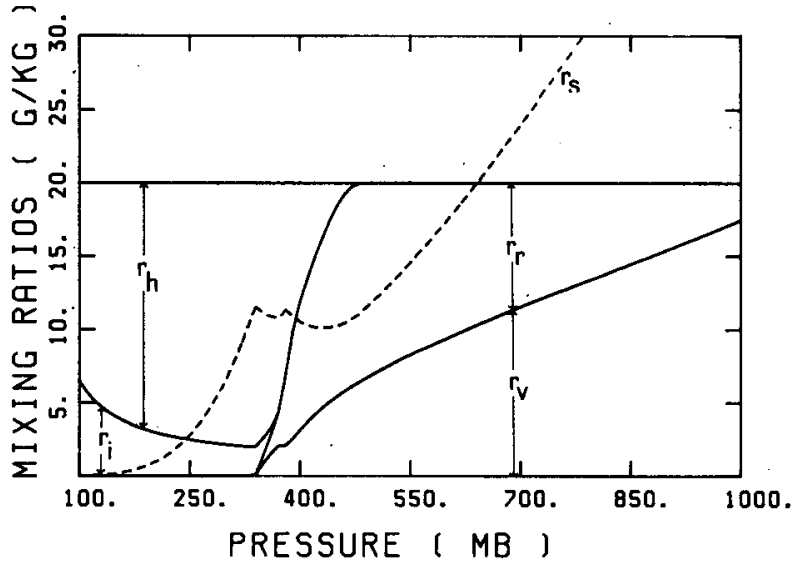

FIG. 3b. As in Fig. 1b except during parcel descent (from left to right) in case II.

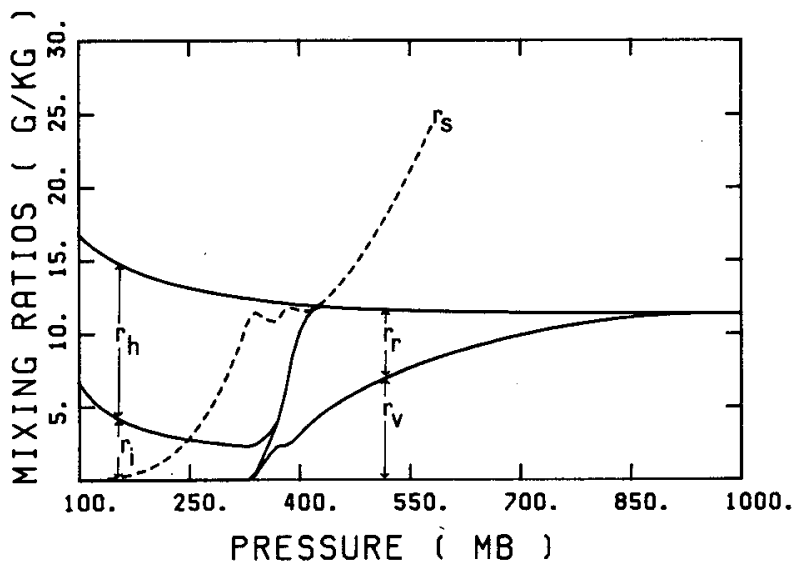

FIG. 4b. As in Fig. 1b except during parcel descent (from left to right) in case III. 


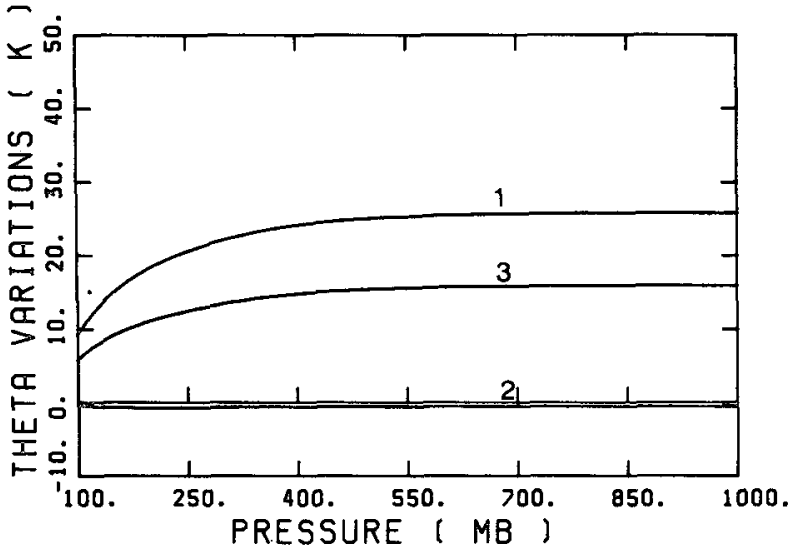

FIG. 5. Variation of 1 , ice-liquid potential temperature (K), 2, wet equivalent potential temperature $(K), 3$, wet equivalent enthalpy (K) (divided by $C_{p d}$ ), as functions of parcel pressure (mb) during parcel descent in case III (from left to right).

following the parcel path. In all circumstances, the variations of WEPT are small and are due only to precipitating particles.

\section{Discussion and conclusions}

This work emphasizes the thermodynamical consequences of such nonreversible phenomena as freezing of supercooled water, evaporation in undersaturated air and precipitation processes. A basic thermodynamical equation is given which involves all the terms considered both by Das (1969) and Tripoli and Cotton (1981). This equation relates the rates of change of temperature, pressure and mixing ratios under all circumstances, even when nonreversible phase changes are involved and equilibrium is not assumed.

Two predictive quantities (WEPT, WEE) are proposed which are analogous to the ice-liquid potential temperature proposed by Tripoli and Cotton for use in numerical models. Any of these quantities, together with the total mixing ratio, can serve as prognostic variables in numerical models instead of the temperature, the total mixing ratio and the water vapor (or droplet) mixing ratio. The use of these predictive quantities avoids the need of a saturation adjustment step and can decrease by one the number of prognostic variables. The actual water vapor or the droplet mixing ratio are simply diagnosed from the new values of the predictive quantity and of the total mixing ratio. All the predictive quantities considered in this work are constant when precipitating particles are absent and their rate of change equations are given whenever precipitating particles appear.

Simple computation with Lagrangian parcel models suggests that the errors are larger when the ice-liquid potential temperature is used than when the wet equivalent potential temperature is used. For both potential temperatures, the errors are larger (up to $5 \mathrm{~K}$ ) when supercooled particles freeze and when precipitating drops or precipitating ice particles evaporate in nonsaturated air. The important advantages of the wet equivalent potential temperature lie in the fact that it can be used in nonhydrostatic flows and its value changes only in the presence of precipitating particles. The largest errors arise when evaporation of particles occurs in nonsaturated air. However, the errors are smaller than when the ice-liquid potential temperature is used. The main drawback of this potential temperature comes from the rather lengthy computational scheme associated with the inversion of its value.

The use of the wet equivalent enthalpy also leads to very small errors, less than $1 \mathbb{K}$, and occurs when supercooled particles freeze. The fact that the rate of change of the pressure appears in the equation for the rate of change of the wet equivalent enthalpy is not a serious drawback for using this predictive variable since, as discussed by Tripoli and Cotton (1981), the coupling between the thermodynamical equation and the continuity equation or the pressure tendency equation exists for any predictive quantity. Furthermore, a nonhydrostatic pressure perturbation at the ground of as much as $10 \mathrm{mb}$ leads to a change in the wet equivalent enthalpy of less than $1 \mathrm{~K}$ which is small compared to the change induced by the precipitation process as shown in Fig. 5. The prediction of temperature, therefore, is not very sensitive to the nonhydrostatic pressure term. It should be accurate enough to estimate the pressure to be used in (22) without taking into account the influence of the unknown change in temperature. The new pressure can then be calculated by using this predicted value of the temperature. Since the wet equivalent enthalpy is mostly quadratic in the independent variables, its inversion can be done on a digital computer with competitive speed. Therefore, the wet equivalent enthalpy should be very useful in the numerical simulation of atmospheric flows by allowing the modeler to take a more complete account of the thermodynamical and dynamical effects of the water species.

Acknowledgments. The author is grateful to Doctor H. Isaka, to Professors H. D. Orville, W. R. Cotton and G. J. Tripoli and to the referees for some useful comments and criticisms on earlier versions of this manuscript.

Many thanks are due to Mrs. C. Baraduc and J. Squarise for their excellent typing and corrections of the manuscript.

\section{APPENDIX}

\section{List of Symbols}

$A_{l v}, A_{i l} \quad$ affinity of vaporization and of melting $\left(\mathrm{J} \mathrm{kg}^{-1}\right)$ 


\begin{tabular}{|c|c|c|}
\hline $\begin{array}{l}C_{i}, C_{l} \\
\quad C_{p d}, C_{p}\end{array}$ & $\begin{array}{l}\text { specific heats at constant pressure of ice, } \\
\text { liquid water, dry air and water vapor } \\
\left(\mathrm{J} \mathrm{K}^{-1} \mathrm{~kg}^{-1}\right)\end{array}$ & $w$ \\
\hline$C_{p t}$ & $\begin{array}{l}\text { mean specific heat at constant pressure of } \\
\text { cloudy air }\left(\mathrm{J} \mathrm{K}^{-1} \mathrm{~kg}^{-1}\right)\end{array}$ & $\begin{array}{l}\gamma \\
\epsilon, \epsilon^{\prime}\end{array}$ \\
\hline$d$ & suffix for dry air & \\
\hline$e_{i}$ & $\begin{array}{l}\text { pressure of water vapor over } \\
\text { irface }(\mathrm{mb})\end{array}$ & \\
\hline$e_{v}, e_{s}$ & $\begin{array}{l}\text { partial pressure of water vapor and its sat- } \\
\text { urated value }(\mathrm{mb})\end{array}$ & \\
\hline & acceleration due to gravity $\left(\mathrm{m} \mathrm{s}^{-2}\right)$ & $\theta, \theta_{i l}, \theta_{q}$ \\
\hline & $-1)$ & $\chi$ \\
\hline$I 1$ & wet equivalent enthalpy $\left(\mathrm{J} \mathrm{kg}^{-1}\right)$ & $\tau$ \\
\hline
\end{tabular}

suffix for ice particles

$l \quad$ suffix for liquid water

$L_{i l} \quad$ latent heat of melting $\left(\mathrm{J} \mathrm{kg}^{-1}\right)$

$L_{l v} \quad$ latent heat of vaporization $\left(\mathrm{J} \mathrm{kg}^{-1}\right)$

$L_{1}, L^{\prime} \quad$ equivalent latent heat due to the vaporization in undersaturated air and to the freezing of supercooled drops $\left(\mathrm{J} \mathrm{kg}^{-1}\right)$

$M_{d}, M_{v}$ molecular weight of dry air and water vapor (kg)

$P, P_{d} \quad$ total pressure and partial pressure of dry air (mb)

$q_{c} \quad$ specific condensate content $\left[=\left(\rho_{l}+\rho_{i}\right) / \rho_{T}\right]$

$r_{T}, r_{c}, r_{l}$, total water mixing ratio, condensed wa-

$r_{r}, r_{i}, \quad$ ter mixing ratio, liquid water mixing

$r_{h}, r_{v} \quad$ ratio, raindrop mixing ratio, ice mixing ratio, hail mixing ratio and water vapor mixing ratio

$R_{d}, R_{v} \quad$ gas constants for dry air and water vapor $\left(\mathrm{J} \mathrm{K}^{-1} \mathrm{~kg}^{-1}\right.$ )

$s_{d}, s_{i}, s_{l}, \quad$ specific entropy of dry air, ice particles, $s_{v} \quad$ liquid water and water vapor $\left(\mathrm{J} \mathrm{K}^{-1}\right.$ $\mathrm{kg}^{-1}$ )

$T, T_{l}, T_{i} \quad$ air temperature, drops temperature and ice particles temperature $(\mathrm{K})$

u velocity vector $\left(\mathrm{m} \mathrm{s}^{-1}\right)$

$v \quad$ suffix for water vapor

$\mathbf{V}_{i}, \mathbf{V}_{h}, \mathbf{V}_{l}$ terminal velocity of ice particles, of hail and of water drops $\left(\mathrm{m} \mathrm{s}^{-1}\right)$ vertical component of the velocity vector $\left(\mathrm{m} \mathrm{s}^{-1}\right)$

vertical coordinate $(\mathrm{m})$

ratio of the specific heats of dry air

ratios of the molecular weight and of the specific heat of water vapor to those of dry air

density of dry air, ice particles, liquid water, water vapor and total density $\left(\mathrm{kg} \mathrm{m}^{-3}\right)$

dry air, ice liquid and wet equivalent potential temperature $(\mathrm{K})$

any variable

relaxation time (s).

\section{REFERENCES}

Betts, A. K., 1973: Non-precipitating cumulus convection and its parameterization. Quart. J. Roy. Meteor. Soc., 99, 178-196.

Bolton, D., 1980: The computation of equivalent potential temperature. Mon. Wea. Rev., 108, 1046-1053.

Brook, R. R., 1978: The influence of water vapor fluctuations on turbulent fluxes. Bound.-Layer Meteor., 15, 481-487.

Das, P., 1969: The thermodynamic equation in cumulus dynamics. J. Atmos. Sci., 26, 399-407.

Dutton, J. A., 1976: The Ceaseless Wind. McGraw Hill, 579 pp.

Iribarne, J. V., and W. L. Godson, 1973: Atmospheric Thermodynamics. Geophys. Astrophys. Monogr., No. 6, Reidel, 222 pp.

Lipps, F. B., and R. S. Hemler, 1980: Another look at the thermodynamic equation for deep convection. Mon. Wea. Rev., 108, 78-84.

Madden, R. A., and F. E. Robitaille, 1970: A comparison of the equivalent potential temperature and the static energy. J. Atmos. Sci., 27, 327-329.

Orville, H. D., and F. J. Kopp, 1977: Numerical simulation of the life history of a hailstorm. J. Atmos. Sci., 34, 1596-1618.

Paluch, I. R., 1979: The entrainment mechanism in Colorado cumuli. J. Atmos. Sci., 36, 2467-2478.

Pruppacher, H. R., and J. D. Klett, 1978: Microphysics of Clouds and Precipitation. Reidel, 714 pp.

Simpson, R. H., 1978: On the computation of equivalent potential temperature. Mon. Wea. Rev., 106, 124-130.

Tag, P. M., 1980: On the diagnosis and conservation of energy during condensation. J. Atmos. Sci., 37, 2347-2351.

Tripoli, G. J., and W. R. Cotton, 1981: The use of ice-liquid water potential temperature as a thermodynamic variable in deep atmospheric models. Mon. Wea. Rev., 109, 1094-1102.

Wilhelmson, R. B., 1977: On the thermodynamic equation for deep convection. Mon. Wea. Rev., 105, 545-549. 